\title{
Gestão do trabalho nas Unidades de Pronto Atendimento: estratégias governamentais e perfil dos profissionais de saúde
}

\section{Workforce management in Emergency Care Units: government strategies and profile of healthcare professionals}

\section{Gestión del trabajo en las unidades de atención de urgencias: estrategias gubernamentales y perfil de los profesionales de salud}

Cristiani Vieira Machado 1

Luciana Dias de Lima 1

Gisele O'Dwyer 1

Carla Lourenço Tavares de Andrade 1

Tatiana Wargas de Faria Baptista 1 Rachel Guimarães Vieira Pitthan 1

Nelson Ibañez 2,3

\section{Resumo}

No final dos anos 2000, a expansão de Unidades de Pronto Atendimento (UPA) no âmbito da política brasileira de atenção às urgências implicou a contratação de muitos profissionais. O artigo analisa as estratégias de gestão do trabalho dos governos e o perfil dos profissionais das UPA no Estado do Rio de Janeiro, que tem o maior número de unidades no país. Os métodos compreenderam: análise documental; entrevistas com gestores; visitas às UPA com aplicação de questionários a coordenadores, médicos e enfermeiros. Os resultados evidenciaram que as estratégias de gestão do trabalho variaram segundo esfera administrativa (estadual ou municipal) e ao longo do tempo. As Organizações Sociais se tornaram as principais contratantes de profissionais nas UPA por propiciarem flexibilidade gerencial. Porém, sobressaíram problemas de seleção e fixação, como a predominância de profissionais jovens pouco experientes e a rotatividade de médicos. A instabilidade associada à contratação por terceiros reforçou a visão da UPA como trabalho temporário.

Emergências; Pessoal de Saúde; Gestão em Saúde; Políticas, Planejamento e Administração em Saúde

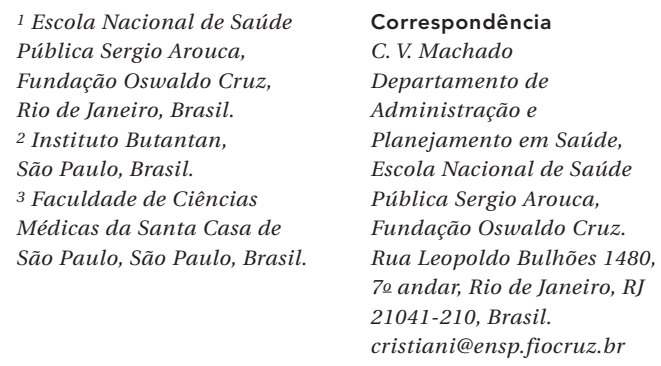

1 Escola Nacional de Saúde Pública Sergio Arouca, Fundação Oswaldo Cruz, Rio de Janeiro, Brasil. 2 Instituto Butantan, São Paulo, Brasil. 3 Faculdade de Ciências Médicas da Santa Casa de São Paulo, São Paulo, Brasil. 


\section{Introdução}

A organização de redes de atenção às urgências nos sistemas de saúde é relevante para a redução da morbimortalidade associada às condições que requerem atendimento imediato. Tal processo envolve a coordenação de diferentes tipos de serviços de saúde com alto grau de interdependência, preparados para atender a uma diversidade de condições, de variada gravidade, em distintos grupos etários 1 .

A decisão política de estruturar uma rede nacional de atenção às urgências exige a expansão de serviços específicos ou a reorganização de serviços existentes, implicando a realização de investimentos em infraestrutura e recursos tecnológicos, bem como a contratação de profissionais de saúde qualificados para esse tipo de atendimento 2 .

No Brasil, nos anos 2000, a atenção às urgências assumiu destaque na agenda federal da saúde. Em que pese o desenho abrangente da Política Nacional de Atenção às Urgências, sua implantação caracterizou-se pela expansão de serviços específicos, marcada por três momentos: 1998-2002 - regulamentação inicial; 20032008 - ênfase nos Serviços de Atendimento Móvel de Urgência (SAMU); a partir de 2009 - ênfase nas Unidades de Pronto Atendimento (UPA), como componente fixo da atenção pré-hospitalar às urgências 3,4 .

A inauguração acelerada das UPA, sob indução normativa e financeira federal, exigiu a contratação de muitos profissionais, expressando desafios gerais da gestão do trabalho no Sistema Único de Saúde (SUS), como dificuldades de fixação, remuneração e desigualdades na distribuição, principalmente de médicos 5,6,7. Isso ocorre em um contexto de aumento das formas de terceirização na gerência de serviços e na contratação dos profissionais de saúde ${ }^{8}$, com destaque para a adoção do modelo Organizações Sociais (OS), proposto no âmbito da Reforma do Estado dos anos $1990{ }^{9}$ e expandido nos anos 200010 .

Aos problemas de gestão do trabalho no SUS somam-se as peculiaridades do trabalho em serviços de urgência. Tais serviços requerem profissionais com alta qualificação técnica, para a garantia de atendimento oportuno e adequado. Além disso, têm uma dinâmica própria de atividades, caracterizada pelo trabalho em ritmo acelerado e intenso, com alto risco de desgaste e sofrimento 11,12 .

O Estado do Rio de Janeiro foi pioneiro na implantação das UPA, iniciada em 2007, antes da regulamentação federal específica. O número de UPA no estado se expandiu nos anos seguin- tes, ultrapassando 60 unidades ao final de 2012, o maior número do país, sendo 50\% localizadas na capital. Outros fatores que tornam o estado um campo rico para estudo são: a existência de unidades vinculadas às esferas estadual e municipal e a adoção de estratégias variadas de lotação de profissionais nas UPA, como o envolvimento de servidores bombeiros e a contratação de OS.

Em face da expansão acelerada das UPA no estado do Rio de Janeiro, cabem as seguintes questões: como os gestores do SUS têm lidado com os desafios da gestão do trabalho nas UPA? Qual é o tipo de vínculo e o perfil dos profissionais predominantes nessas unidades? Que fatores influenciam as estratégias adotadas e quais as suas possíveis repercussões sobre a gestão do trabalho em saúde?

Este artigo apresenta parte dos resultados de uma pesquisa sobre a implementação das UPA no Estado do Rio de Janeiro, tendo o objetivo de analisar as estratégias governamentais de gestão do trabalho, as condições de vínculo e o perfil dos profissionais de saúde atuantes nas UPA no estado.

\section{Metodologia}

A pesquisa compreendeu dois eixos de análise: a identificação das estratégias de gestão do trabalho adotadas para as UPA; as condições de inserção e o perfil dos profissionais de saúde nas UPA.

Em face do expressivo número de UPA no Estado do Rio de Janeiro e de sua presença em municípios de diferentes regiões, optou-se pela definição de critério amostral para seleção das UPA a serem investigadas. A amostra foi calculada a partir do universo de 42 UPA localizadas no estado, que estavam cadastradas junto ao Ministério da Saúde e apresentavam mais de um ano de funcionamento em 2012, quando se iniciou a pesquisa. Tais UPA apresentavam a seguinte distribuição: 22 na capital, 9 no entorno metropolitano e 11 nos municípios do interior.

Para o cálculo do tamanho da amostra foram considerados os parâmetros: valor da abscissa da tabela da Normal de $90 \%$ (bilateral), proporção (UPA existentes no Estado do Rio de Janeiro) de 0,5 e erro amostral de $15 \%$. O critério de seleção da amostra foi do tipo proporcional ao tamanho nos estratos previamente definidos. A seleção das UPA foi realizada por amostragem sistemática em cada estrato. Foram selecionadas 19 UPA, localizadas em dez municípios, assim distribuídas: 10 na capital, 4 no entorno metropolitano e 5 no interior.

O trabalho de campo foi desenvolvido entre novembro de 2012 e janeiro de 2013. Em um pri- 
meiro momento, os métodos envolveram: análise de documentos federais, estaduais e municipais (normas, portarias, contratos de gestão, relatórios); análise de dados secundários; realização de 41 entrevistas semiestruturadas com gestores envolvidos com a política de urgências na esfera federal, estadual e nos dez municípios. Os entrevistados foram selecionados segundo cargo ocupado (Secretários de Atenção à Saúde, Secretários ou Subsecretários de Saúde, Coordenadores da rede de atenção) e responsabilidade sobre a política de atenção às urgências/UPA.

Os roteiros de entrevistas compreenderam questões referentes à implementação das UPA, incluindo: estratégias de gestão do trabalho; visão dos dirigentes sobre as formas de inserção; perfil dos profissionais e desafios da gestão do trabalho nas UPA. Após transcrição, procedeuse à análise de conteúdo temático das entrevistas, cujos resultados foram cotejados com outras fontes de pesquisa.

$\mathrm{Na}$ apresentação dos resultados, em caso de citação direta de falas, os entrevistados foram identificados pela esfera de governo a que se vinculam e, no caso dos municípios, tipo de localização no estado, conformando os grupos: Gestor Federal (GF); Gestor Estadual (GE); Gestor Municipal-Capital (GMC); Gestor Municipal-Entorno Metropolitano (GMEM); Gestor Municipal-Interior (GMI). Em cada grupo, as entrevistas foram numeradas sequencialmente $(1,2,3 \ldots)$. O cargo de cada entrevistado não foi indicado para evitar a sua identificação.

Em um segundo momento, foram feitas visitas às 19 UPA da amostra, com preenchimento de diário de campo e aplicação in loco de 76 questionários estruturados junto a: 19 coordenadores, 38 médicos e 19 enfermeiros. Os médicos (dois por unidade) e enfermeiros (um por unidade) foram escolhidos aleatoriamente, de acordo com sua disponibilidade no dia da visita.

Os questionários compreendiam blocos relativos a: (i) modelo de gestão da UPA; (ii) gestão do trabalho na UPA (forma de seleção e contratação, tipo de vínculo, carga horária, remuneração); (iii) perfil dos profissionais (sexo, idade, perfil e tempo de formação, experiência prévia, entre outros); (iv) oferta de serviços e assistência prestada; e (v) articulação com a rede.

Para fins deste artigo, são consideradas as respostas relativas aos segundo e terceiro blo$\cos$. Assinale-se que algumas foram respondidas apenas pelos coordenadores (19 respondentes); outras foram respondidas por coordenadores, médicos e enfermeiros (76 respondentes).

As respostas aos questionários foram digitadas no programa Epi Info (Centers for Disease Control and Prevention, Atlanta, Estados Uni- dos). Os dados foram exportados para o programa IBM-SPSS (IBM Corp., Armonk, Estados Unidos) e submetidos à tabulação e análise.

O projeto de pesquisa foi aprovado por Comitê de Ética em Pesquisa (parecer 158.346 de 2012).

\section{Resultados}

Estratégias de gestão do trabalho para as UPA: formas de contratação, fixação, formação e capacitação profissional

As UPA no Rio de Janeiro no momento do estudo estavam distribuídas por várias regiões do estado e apresentavam perfil diversificado no que concerne à localização, ano de inauguração, vinculação à esfera estadual ou municipal e modelo de gestão (administração direta ou OS). A Tabela 1 resume características das UPA da amostra - representativa para o estado - que têm implicações para a gestão do trabalho.

Em primeiro lugar, ressalte-se que, no momento da pesquisa, $57,9 \%$ das UPA estavam ligadas à esfera estadual, indicando a importância do estado na expansão dessas unidades, principalmente na região metropolitana, que comporta as primeiras UPA, inauguradas em 2007 e 2008. Mesmo municípios que implantaram UPA sob gestão própria a partir de 2009 referiram a influência do estado, pioneiro nessa estratégia, além da indução normativa e financeira federal.

Em segundo lugar, mais de um terço das UPA localizadas no estado estava sob gestão de diferentes OS $(36,8 \%)$, principalmente as vinculadas ao Município do Rio de Janeiro. Cabe destacar que o trabalho de campo ocorreu em um contexto de transição, em que a maior parte das UPA estaduais estavam passando da administração direta para a gestão de OS.

Portanto, a vinculação estadual ou municipal das UPA teve implicações para o modelo de gestão das unidades e para as estratégias de gestão de trabalho, particularmente no que se refere às formas de contratação e esforços de fixação de profissionais.

As UPA estaduais foram implantadas inicialmente sob o modelo de administração direta, predominante entre 2007 e 2012. Nesse período foram experimentadas diferentes formas de contratação dos profissionais de saúde. A opção inicial do governo estadual foi lotar os servidores militares bombeiros nas UPA, o que teve relação com a unificação da Defesa Civil e da Saúde na mesma secretaria estadual de 2007 a 2010 e com a experiência prévia dos bombei- 
Unidades de Pronto Atendimento (UPA) estudadas, segundo esfera administrativa, ano de inauguração e modalidade de gestão. Estado do Rio de Janeiro, Brasil, 2012-2013.

\begin{tabular}{|c|c|c|c|}
\hline UPA & Ano de inauguração & Esfera administrativa & Modelo de gestão \\
\hline \multicolumn{4}{|c|}{ Município do Rio de Janeiro } \\
\hline 1 & 2007 & Estadual & Administração direta, em transição para OS \\
\hline 2 & 2007 & Estadual & Administração direta, em transição para OS \\
\hline 3 & 2007 & Estadual & Administração direta, em transição para OS \\
\hline 4 & 2008 & Estadual & Administração direta, em transição para OS \\
\hline 5 & 2008 & Estadual & Administração direta \\
\hline 6 & 2009 & Municipal & OS \\
\hline 7 & 2010 & Municipal (iniciativa estadual) & OS \\
\hline 8 & 2010 & Municipal & OS \\
\hline 9 & 2010 & Estadual & Administração direta, em transição para OS \\
\hline 10 & 2010 & Municipal & OS \\
\hline \multicolumn{4}{|c|}{ Municípios do entorno metropolitano } \\
\hline 11 & 2008 & Estadual & Administração direta \\
\hline 12 & 2008 & Estadual & OS \\
\hline 13 & 2009 & Estadual & OS \\
\hline 14 & 2010 & Estadual & Administração direta, em transição para OS \\
\hline \multicolumn{4}{|c|}{ Municípios do interior } \\
\hline 15 & 2009 & Municipal (iniciativa estadual) & Administração direta \\
\hline 16 & 2009 & Municipal (iniciativa estadual) & Administração direta \\
\hline 17 & 2010 & Municipal (iniciativa estadual) & Administração direta \\
\hline 18 & 2010 & Municipal (iniciativa estadual) & Administração direta, em transição para OS \\
\hline 19 & 2010 & Estadual & Administração direta, em transição para OS \\
\hline
\end{tabular}

OS: Organização Social.

ros no SAMU. Assim, os bombeiros assumiram funções de gerência e assistência nas UPA no referido período.

Além disso, os entrevistados referiram a expectativa de que, em função da disciplina militar, se conseguisse manter os médicos bombeiros em UPA sediadas em locais de difícil fixação, na periferia da cidade ou em áreas de maior violência urbana. No entanto, mesmo nesses casos se observaram dificuldades de fixação:

"Essa dificuldade do vazio sanitário a gente tentou enfrentar [...] fez um concurso para bombeiros. Muitos pediram baixa porque o volume de atendimento era muito grande, o local era difícil" (GE 1).

No momento da pesquisa (2012-2013), as UPA estaduais expressavam um movimento de substituição dos servidores bombeiros por profissionais contratados por terceiros, compreendendo um quadro de profissionais sob vínculos variados: bombeiros (parte dos coordenadores, médicos e enfermeiros), profissionais contratados por cooperativas ou OS (parte dos profissionais de nível superior, técnicos de enfermagem) e profissionais vinculados a serviços terceirizados (análises clínicas, radiologia, limpeza).

A mudança da gestão significava a transferência de responsabilidades de gerência das unidades e contratação de profissionais de saúde para as OS. Conforme os entrevistados, essa opção se deu pelo esgotamento das tentativas anteriores e limites em assegurar a fixação de profissionais:

"A gente foi tentando muitas estratégias para fixar [...] por isso, a dificuldade em conseguir médico e o engessamento salarial [...] surge a gestão por OS, que pode oferecer um vínculo mais sólido, o CLT para o funcionário, cobrar o que é exigido" (GE 2).

Já o Município do Rio de Janeiro desde 2009 vinha inaugurando UPA próprias sob o modelo de contratação de OS, também adotado para a expansão das Clínicas da Família na Atenção Básica. Os dirigentes municipais justificaram a opção pelas OS com base nos argumentos: impossibilidade de suprir a rápida expansão da oferta de serviços com servidores concursados; restrições da Lei de Responsabilidade Fiscal aos gastos com pessoal; dificuldades de contratar médicos 
e limites da gestão do trabalho pelas regras da administração pública direta:

“Isso não é opção, é falta de opção. Não tem como hoje, o município ter feito a expansão dos serviços de saúde que fez só com a administração direta" (GMC 1).

“...por conta de você crescer muito e ter a lei da responsabilidade fiscal, que limita essa questão de salário, de contratação” (GMC 2).

"Vejo como maior obstáculo na concepção das políticas de saúde como um todo e especificamente, na política de urgência e emergência dos municípios, a questão dos recursos humanos, da gestão de pessoas. Não se trata de uma posição ideológica contra ou a favor do funcionalismo público [...] faltam mecanismos que permitam uma adequada gestão de pessoas. A meu ver, a legislação que ampara o funcionalismo público é paternalista" (GMC 3).

“A legislação da organização social foi uma inovação. Acho que é teste ainda. Foi o que possibilitou fazer funcionar, terceirizar para garantir os recursos humanos, principalmente o cumprimento da carga horária" (GMC 4).

Apesar de o modelo OS ser adotado no município para outros tipos de unidades, a natureza do trabalho em urgência também foi apontada como dificuldade adicional para fixar profissionais nas UPA:

"Nesse cenário de pronto-atendimento, isso é mais crítico ainda. É difícil o indivíduo assumir um compromisso aos 20 poucos, 30 anos de idade e dizer que ele vai ficar no pronto-atendimento até se aposentar, porque não vai, não aguenta" (GMC 1).

Na perspectiva dos gestores, a contratação de OS traria como vantagens para a gestão do trabalho: contratações e substituições de profissionais de forma ágil, por processos seletivos simplificados (em geral, análise de currículos e entrevistas); remuneração em valores de mercado, atraentes para os médicos; estabelecimento de metas e controles sobre os profissionais, com possibilidade de demissão por insuficiência de desempenho.

A escassez e as dificuldades em lotar médicos foram enfatizadas pelos entrevistados:

"O problema é a questão dos profissionais de saúde, sobretudo, dos médicos [...] Não tem capacidade de remuneração que atraia, uma força de trabalho de reserva no mercado para suprir, porque tem escassez relativa de médicos. [...] Quando comparamos com Inglaterra, França, Espanha, temos menos médicos e, além disso, mal distribuídos" (GF 1).

“É inviável pensar em saúde, se não pensar em médico [...] é portaria aumentando cobertura de Atenção Básica, criando UPA, leito disso, daquilo, e não temos, nem em quantidade, nem em qualidade, os profissionais de saúde que precisamos" (GE 3).

Ressalte-se que os problemas de fixação dos profissionais foram mais graves na periferia metropolitana e nas áreas da capital de baixo nível socioeconômico, carentes de serviços e marcadas pela violência:

"Acho que tem que regionalizar. Tem que investir mais aqui, incentivar o médico a se fixar na Baixada. É um problema. Nós temos poucos médicos residentes na Baixada Fluminense" (GMEM 1).

"Tem dia que não tem clínico, tem dia que não tem pediatra. Pediatra então, ficamos um tempo enorme com duas UPA sem pediatria [...] a dificuldade ainda é a irregularidade de profissional" (GMEM 2).

Porém, os entrevistados divergiram em relação à possibilidade de a contratação de OS atenuar os problemas relativos à fixação de profissionais. Enquanto alguns defenderam que as OS seriam uma solução parcial para as dificuldades de gestão do trabalho, outros advertiram que a alta rotatividade poderia ser agravada pela instabilidade dos vínculos terceirizados, mudança periódica da OS contratante e variedade na remuneração entre municípios.

"Você não consegue fixar o profissional. Porque todo mundo contratado, não tem vínculo formal. Então você não fixa profissional. Eu pago cinco mil, se ela pagar cinco e quinhentos, [o profissional] vai embora" (GE 4).

"Também enfrentamos uma dificuldade grande quando houve mudanças. Em um primeiro momento estavam lotados só militares. Então, a gente não tinha déficit de profissional, porque o militar não faltava ao plantão de jeito nenhum. Depois entrou cooperativa, não sei exatamente o que entrou, mas outro vínculo [...] passou a ter buraco de profissional" (GMEM 2).

No que concerne aos municípios em regiões do interior do estado, no momento da pesquisa, a maior parte das UPA estava ligada à esfera municipal, sob administração direta. Porém, várias delas haviam sido inauguradas sob gestão estadual e foram transferidas aos municípios posteriormente. Assim, as formas de contratação de profissionais eram variadas, compreendendo estatutários e formas diversas de terceirização, a depender do município e da trajetória da unidade. Houve preocupação com a instabilidade associada à terceirização da gestão e à diversidade de tipos de contratação e vínculos profissionais:

"Tinha que ter concurso sim, mas concurso celetista [...] se todos que estão na OS são celetistas, porque no serviço público não podemos ter celetista? A gente não consegue, aí começa as aberrações: 
OS, OSCIP ... São Paulo já pagou por isso e aqui está começando [...] no estado um é OS, um é OSCIP, fundação, cooperativa" (GMI 1).

Também se relatou a dificuldade de lotação e fixação dos médicos, bem como a rotatividade de médicos entre unidades de saúde. Em alguns municípios, a inauguração de UPA levou a fechamento de unidades de pronto atendimento pré-existentes ou ao deslocamento de médicos de outros tipos de serviços para as UPA que, no entanto, muitas vezes não lograram fixar os profissionais.

De forma associada à instabilidade do vínculo predominante nas OS, outro motivo apontado para a rotatividade foi o predomínio de profissionais jovens que buscam emprego temporário nas UPA:

"Esses meninos formam, vão trabalhar uns meses em urgência, juntam um dinheiro e saem... não cria vínculo, precisa construir vínculo" (GMI 1).

No que concerne à questão da formação e capacitação profissional, foram referidos problemas similares pelos entrevistados dos diferentes grupos, incluindo limites da formação universitária, em geral e para a atenção às urgências:

"Estudos têm sido feitos [...] para entender a necessidade de aumentar o número de cursos de medicina, para que tenhamos mais médicos. Mas aí vem aquela interrogação, que qualidade que você vai entregar [...] o grande desafio hoje, mais até do que o financiamento, é a formação de recursos humanos, especialmente médicos" (GE 3).

“...um esforço enorme de qualificação tem que ser feito, que não é restrito ao pronto-atendimento. Mas nesse cenário que lida com possibilidade de risco à vida e risco de morte, lógico que isso toma uma proporção maior [...] a questão da qualificação é mais profunda do que ser seleção pública de OS ou concurso público. A qualificação profissional passa pela capacidade de formar bons profissionais. Eu acho que o cerne da questão é: que profissional está saindo da graduação hoje? Que profissional está saindo dos programas de residência médica?” (GMC 1).

"O nível profissional dos médicos, para não falar só dos médicos, dos enfermeiros... tem muitos médicos recém-formados com dificuldade até na interpretação de eletro..." (GMI 2).

Diante desse cenário, a oferta das capacitações específicas para a atuação em urgências foi citada pelos gestores estaduais e municipais. No caso das UPA estaduais, alguns entrevistados destacaram os cursos promovidos no período de participação da Corporação dos Bombeiros, dada a sua experiência com esse tipo de capacitação.

Diversos aspectos identificados na primeira fase da pesquisa, por meio da análise de docu- mentos, dados oficiais e entrevistas, foram corroborados ou aprofundados na segunda fase da pesquisa, de visita às UPA e entrevistas com coordenadores de unidades e profissionais, cujos resultados são apresentados a seguir.

Os trabalhadores nas UPA: tipo de vínculo, perfil e formação dos profissionais de saúde

No quadro de profissionais das UPA visitadas predominavam técnicos de enfermagem, enfermeiros, clínicos gerais, pediatras, odontólogos e assistentes sociais, sendo o número programado de profissionais em geral suficiente, na opinião dos entrevistados. Por outro lado, 68,4\% dos coordenadores relataram dificuldades de recrutamento e fixação de profissionais, principalmente de pediatras e clínicos gerais. Tais dificuldades foram maiores para as UPA localizadas no entorno metropolitano ou em comunidades carentes da capital.

A Figura 1 apresenta a distribuição das UPA da amostra segundo tipo de vínculo profissional predominante. Observa-se que $74 \%$ dos coordenadores das UPA afirmaram que a maior parte dos profissionais de sua unidade apresentava vínculos formais regidos pela Consolidação das Leis do Trabalho (CLT), sendo comum a convivência de vínculos distintos em uma mesma unidade.

Em relação ao tipo de contratante predominante, $57,9 \%$ dos coordenadores referiram que as OS eram os principais contratantes diretos dos profissionais na sua UPA (Figura 2). Mesmo nas UPA sob administração direta, parte expressiva das contratações dos profissionais já era efetivada por meio de terceiros, incluindo OS, fundações ou Organizações Não-Governamentais.

Quanto aos mecanismos de seleção de profissionais, segundo a maioria dos coordenadores, os médicos em geral foram selecionados por meio de entrevistas e análises de currículo, sendo que três coordenadores mencionaram não haver processo formal de seleção desses profissionais. A contratação de médicos por concurso público foi rara, referida apenas no caso dos médicos bombeiros das UPA estaduais, que no momento da pesquisa já eram minoria. No caso dos enfermeiros, os mecanismos utilizados para contratação foram diversificados, eventualmente envolvendo a realização de provas escritas e práticas.

A Tabela 2 descreve as características de perfil dos coordenadores, médicos e enfermeiros entrevistados, considerando: sexo; faixa etária; formação/capacitação; experiência prévia em urgência ou emergência; carga horária e proporção da remuneração aferida na UPA na renda total do profissional. 
Figura 1

Distribuição das Unidades de Pronto Atendimento (UPA), por tipo de vínculo dos profissionais predominante. Estado do Rio de Janeiro, Brasil, 2012-2013.

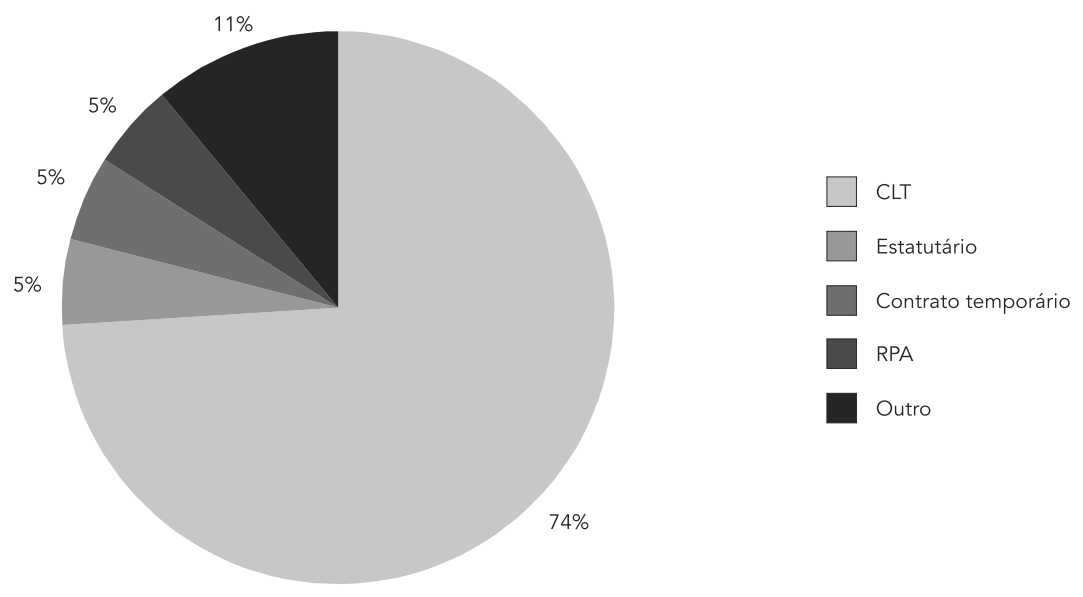

Fonte: questionários aplicados aos coordenadores de UPA ( $\mathrm{n}=19$ questionários)

CLT: Consolidação das Leis do Trabalho; RPA: Recibo de Pagamento a Autônomo.

Figura 2

Distribuição das Unidades de Pronto Atendimento (UPA), por tipo de contratante predominante. Estado do Rio de Janeiro, Brasil, 2012-2013

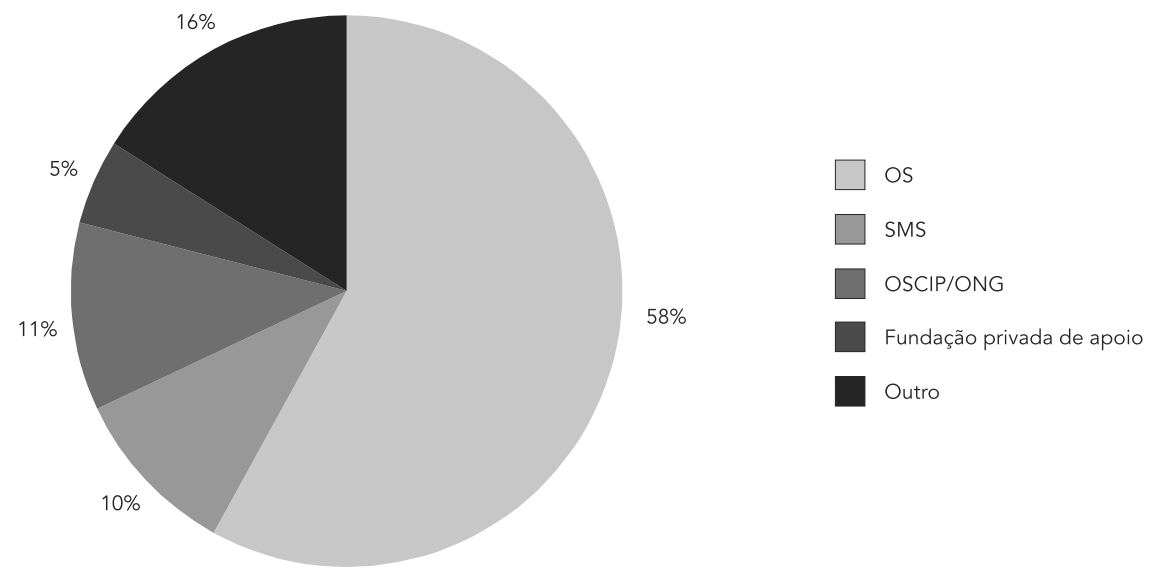

Fonte: questionários aplicados aos coordenadores de UPA ( $n$ = 19 questionários).

ONG: Organização Não Governamental; OS: Organizações Sociais; OSCIP: Organização da Sociedade Civil de Interesse Público; SMS: Secretaria Municipal de Saúde. 
Distribuição dos coordenadores, médicos e enfermeiros entrevistados, segundo características de perfil pessoal, formação, experiência prévia e inserção profissional nas Unidades de Pronto Atendimento (UPA). Estado do Rio de Janeiro, Brasil, 2012-2013.

\begin{tabular}{|c|c|c|c|}
\hline Variáveis/Descrição & $\begin{array}{l}\text { Coordenadores (\%) } \\
\qquad(n=19)\end{array}$ & $\begin{array}{c}\text { Médicos (\%) } \\
\quad(n=38)\end{array}$ & $\begin{array}{l}\text { Enfermeiros (\%) } \\
\qquad(n=19)\end{array}$ \\
\hline \multicolumn{4}{|l|}{ Sexo } \\
\hline Masculino & 63,2 & 44,7 & 15,8 \\
\hline Feminino & 36,8 & 55,3 & 84,2 \\
\hline \multicolumn{4}{|l|}{ Faixa etária (em anos) } \\
\hline Até 30 & 26,3 & 47,4 & 63,2 \\
\hline $31-40$ & 26,3 & 36,8 & 26,3 \\
\hline $41-50$ & 42,1 & 7,9 & 10,5 \\
\hline $51-60$ & 5,3 & 2,6 & 0,0 \\
\hline Mais de 60 & 0,0 & 5,3 & 0,0 \\
\hline \multicolumn{4}{|c|}{ Natureza jurídica do curso de graduação } \\
\hline Público & 36,8 & 28,9 & 21,1 \\
\hline Privado & 63,2 & 71,1 & 78,9 \\
\hline \multicolumn{4}{|c|}{ Tempo de conclusão da graduação (em anos) } \\
\hline Até 2 & 0,0 & 34,3 & 10,5 \\
\hline $2-8$ & 36,8 & 28,9 & 21,1 \\
\hline Mais de 8 & 57,9 & 34,0 & 21,1 \\
\hline Sem informação & 5,3 & 0,0 & 0,0 \\
\hline \multicolumn{4}{|c|}{ Especialização ou Residência } \\
\hline $\operatorname{Sim}$ & 100,0 & 57,9 & 42,1 \\
\hline \multicolumn{4}{|c|}{ Curso de capacitação em Urgência/Emergência } \\
\hline Sim & 78,9 & 78,9 & 84,2 \\
\hline \multicolumn{4}{|c|}{ Experiência prévia em Urgência/Emergência } \\
\hline Sim & 94,7 & 73,7 & 52,6 \\
\hline \multicolumn{4}{|l|}{ Tipo de vínculo na UPA } \\
\hline CLT & 73,7 & 57,9 & 47,4 \\
\hline Estatutário & 26,3 & 21,1 & 21,1 \\
\hline Outros & 0,0 & 21,0 & 31,5 \\
\hline \multicolumn{4}{|c|}{ Carga horária contratual na UPA (em horas semanais) } \\
\hline 12 & 0,0 & 15,8 & 0,0 \\
\hline 24 & 21,1 & 63,2 & 21,1 \\
\hline 40 & 52,6 & 2,6 & 42,1 \\
\hline Outra & 26,3 & 18,4 & 36,8 \\
\hline \multicolumn{4}{|c|}{ Percentual da remuneração da UPA na renda total } \\
\hline Até 50 & 47,4 & 70,3 & 42,1 \\
\hline Mais de 50 & 52,7 & 29,7 & 57,9 \\
\hline
\end{tabular}

Fonte: questionários aplicados aos Coordenadores, Médicos e Enfermeiros das UPA da amostra ( $n$ UPA = 19;

n questionários $=76$ ).

CLT: Consolidação das Leis do Trabalho.

Nos cargos de coordenação das unidades de pronto atendimento, observa-se predominância de médicos $(84,2 \%)$, do sexo masculino $(63,2 \%)$ e de profissionais de faixas etárias intermediárias (68,4\% entre 31 e 50 anos). Já entre médicos e enfermeiros predominaram as mulheres e as faixas etárias mais jovens; $47,4 \%$ dos médicos e $63,2 \%$ dos enfermeiros apresentavam até 30 anos.
A formação em universidades privadas predominou nos três grupos. O tempo de conclusão de graduação foi maior para os coordenadores: $57,9 \%$ haviam se formado há mais de oito anos. Já a presença de recém-formados (conclusão da graduação há menos de dois anos) foi marcante entre os médicos $(34,3 \%)$, sendo que um quinto dos entrevistados havia se formado há menos de um ano. 
Quanto à pós-graduação, todos os coordenadores de UPA relataram ao menos um curso de especialização ou residência, a maioria de perfil clínico. Entre os médicos, a proporção de profissionais com especialização ou residência concluída foi de somente $57,9 \%$, com destaque para as especialidades: pediatria, cardiologia, clínica médica/medicina interna e cirurgia. Entre os enfermeiros, apenas $42,1 \%$ referiram curso de especialização.

Ressalte-se que a maioria dos entrevistados relatou a realização de alguma capacitação em atenção às urgências após a conclusão da graduação - 78,9\% dos coordenadores e médicos; $84,2 \%$ dos enfermeiros -, com destaque para cursos de curta duração de "suporte de vida" dirigidos a trauma (Advanced Trauma Life Support - ATLS) ou cardiologia (Advanced Cardiovascular Life Support-ACLS).

No que concerne à experiência profissional, 94,7\% dos coordenadores já tinham experiência em atenção às urgências/emergências anterior à inserção na UPA. Entre os médicos, essa proporção foi de $73,7 \%$; e entre os enfermeiros, de $52,6 \%$. O tipo mais frequente de experiência prévia relatada pelos três grupos foi em emergências hospitalares, mas também foram referidas experiências em outras UPA e no SAMU.
A carga horária cumprida pela maioria dos coordenadores foi de 40 horas semanais $(52,6 \%)$, por meio de rotina de trabalho diurna. Já para os médicos, predominou a carga de 24 horas $(63,2 \%)$, exercida por meio de um ou dois plantões. No caso dos enfermeiros, as cargas horárias mais frequentes foram 40 e 24 horas semanais.

A proporção da renda aferida na UPA na renda total dos profissionais foi mais importante para os coordenadores e enfermeiros, expressando o comprometimento de carga horária, embora muitos tivessem outras fontes de renda. Entre os médicos, para mais de $70 \%$, a renda aferida na UPA representava menos da metade de sua renda total.

Por fim, a Figura 3 apresenta a distribuição dos médicos e enfermeiros quanto aos fatores que os motivaram a trabalhar nas UPA. Os médicos destacaram principalmente: proximidade do domicílio (39,5\%) e boa remuneração $(31,6 \%)$, seguidos por oportunidade de primeiro emprego, ganho de experiência profissional e afinidade com o trabalho em urgências (cada um citado por 26,3\%). Já os enfermeiros apontaram: ganho de experiência profissional $(57,9 \%)$, proximidade do domicílio $(42,1 \%)$ e afinidade com o tema das emergências $(42,1 \%)$.

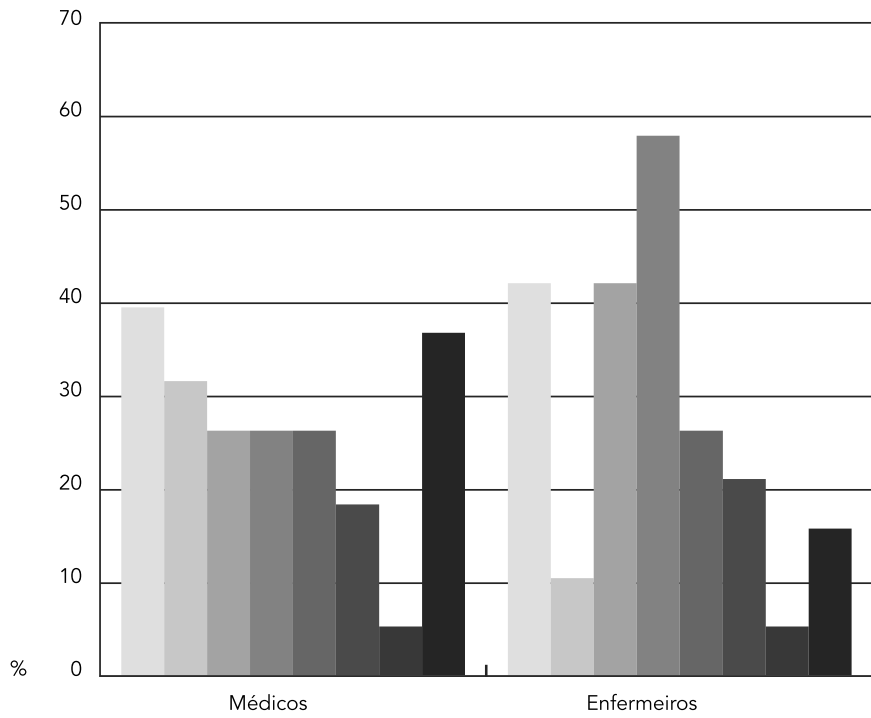

Proximidade do domicílio

Boa remuneração

Afinidade com trabalho em urgências

Ganho de experiência profissiona

Oportunidade de primeiro emprego

Flexibilidade de horário

Flexibilidade de exigência de formação

Outros

Fonte: questionários aplicados aos médicos e enfermeiros das UPA da amostra ( $\mathrm{n}$ médicos = 38; $\mathrm{n}$ enfermeiros = 19). 
Ressalte-se que a maioria dos médicos $(63,2 \%)$ e dos enfermeiros $(52,6 \%)$ considerava a UPA como um emprego por tempo determinado, sendo frequentemente apontada como motivo, pelos dois grupos, a insegurança relacionada à contratação por OS. Entre os médicos, destacou-se também o desejo de realizar uma residência.

\section{Discussão}

O Estado do Rio de Janeiro foi pioneiro na implantação das UPA desde 2007 e, em 2012, apresentava o maior número dessas unidades no país.

A pesquisa mostrou que a rápida expansão das UPA no estado teve repercussões para as estratégias de gestão do trabalho em saúde, que variaram segundo a esfera administrativa da UPA e no tempo. Assim, o governo do estado, no momento inicial de implantação das UPA estaduais (2007-2012), privilegiou a inserção de servidores bombeiros nas funções gerenciais e parte das assistenciais. As UPA ligadas ao município do Rio foram inauguradas a partir de 2009 sob gestão de OS, que assumiram a contratação dos profissionais de saúde. As UPA vinculadas às secretarias municipais do interior em geral foram inauguradas sob a administração direta, sendo frequente a coexistência de formas variadas de contratação de profissionais.

No entanto, em 2012-2013, no momento da pesquisa, sobressaía certa convergência entre as UPA estaduais e municipais, no sentido da expansão da contratação das OS para a gerência de unidades e profissionais de saúde. Nesse contexto, a maior parte dos entrevistados nas secretarias e nas unidades de saúde indicou que as OS já eram as principais contratantes diretas dos profissionais nas UPA, que em sua maioria apresentavam vínculo trabalhista regido pela CLT. Relatou-se com frequência a coexistência de diferentes tipos de vínculos nas unidades.

Tal resultado é consoante com a expansão das novas modalidades de gestão e contratação de profissionais no SUS desde os anos 1990, relacionada com processos de reforma do Estado e de reestruturação do trabalho ${ }^{8}$. A agenda hegemônica de reformas na Administração Pública brasileira naquela década enfatizava o papel regulador do Estado e a adoção de estratégias e instrumentos inspirados na "nova gestão pública", como parcerias público-privadas, flexibilização gerencial, mecanismos contratuais e avaliação por resultados 13 .

Em meados da década, o modelo OS foi proposto pelo Governo Federal para a prestação de atividades "não-exclusivas" de estado 9 . O Estado de São Paulo foi pioneiro na contratação de OS para a prestação de serviços hospitalares 14,15,16, sendo o modelo posteriormente expandido para outros tipos de unidades de saúde.

No Estado do Rio de Janeiro, nos anos 1990, a possibilidade de adoção do modelo OS em hospitais foi debatida, porém predominou a combinação de administração direta com outros modelos, como fundações privadas de apoio e cooperativas ${ }^{17}$. Nas unidades municipais de atenção básica, predominava o modelo de administração direta com contratação de servidores estatutários, associado a experiências alternativas de contratação pontuais, em áreas de difícil fixação (por exemplo, por meio de convênios com associações de moradores).

A partir de meados dos anos 2000, o modelo OS se expandiu aceleradamente na saúde no território nacional. Dados do Instituto Brasileiro de Geografia e Estatística (IBGE) apontam que, em 2013, a contratação de serviços de saúde por meio de OS era feita por 17 estados 18 e por mais de 1.216 municípios brasileiros, ou 21,8\% do total do país, sendo essa proporção de mais de $50 \%$ entre os municípios com mais de $100 \mathrm{mil}$ habitantes 19 .

Somente a partir de 2009, observa-se no Estado do Rio de Janeiro expressiva expansão da contratação de OS, impulsionada pela opção do Município do Rio de Janeiro de adotar tal modelo, associado à ampliação da cobertura da Estratégia Saúde da Família na capital 20 e à expansão das UPA, objeto desta pesquisa.

As razões relatadas pelos gestores entrevistados para a contratação das OS, em contraposição à administração direta, se associaram a três argumentos principais: a necessidade de rápida expansão de serviços em cenário de constrangimentos legais e financeiros ao aumento do funcionalismo; a maior flexibilidade para contratar, remunerar e demitir; a dificuldade de fixação de profissionais em serviços de saúde localizados em áreas de vulnerabilidade social.

Acrescente-se um quarto argumento levantado por alguns entrevistados, relativo à natureza do trabalho de atenção às urgências, que dificultaria a fixação de profissionais. Tal argumento encontra respaldo em estudos que destacam o alto risco de desgaste e sofrimento relacionado ao caráter e ao ritmo intenso de trabalho nesses serviços 11,12. Ressalte-se ainda que diferentes expectativas de usuários e profissionais de saúde quanto ao atendimento em urgência podem gerar tensões e insatisfação em ambos os lados 21 .

No entanto, houve visões variadas entre os entrevistados quanto à possibilidade do modelo 
OS resolver os problemas de contratação e fixação de profissionais. Se por um lado as OS propiciaram agilidade de contratação e melhor remuneração, por outro os problemas de fixação e formação dos profissionais sobressaíram. Alguns entrevistados relataram que as OS gerariam um clima de instabilidade que dificultaria o vínculo dos profissionais com o serviço. Isso ajudaria a explicar a expressiva proporção de médicos e enfermeiros que indicaram a UPA como um trabalho temporário e citaram motivações práticas para trabalhar na unidade, como proximidade ao domicílio, possibilidade de primeiro emprego e ganho de experiência profissional.

Os dois últimos motivos também se relacionam à alta proporção de profissionais jovens nas UPA. Parcela expressiva dos médicos e enfermeiros havia se graduado há menos de dois anos e não havia realizado curso de especialização nem de residência, o que prejudicaria a qualidade da assistência, segundo alguns entrevistados. A preponderância de profissionais formados em faculdades privadas, por sua vez, é explicada pela maior oferta de vagas de Medicina e Enfermagem nesses cursos 22 .

Dificuldades relacionadas à formação curricular também foram apontadas para explicar limites de adequação de perfil e de fixação dos profissionais em serviços do SUS, em sintonia com a literatura 23 . Ainda que esse problema não se restrinja às urgências, o déficit de formação nessa área foi reconhecido por gestores e profissionais, justificando a oferta de cursos de curta duração e a aposta no ganho de experiência in loco.

Por fim, a permanência de profissionais em serviços de urgências requer, além de preparo técnico, certa identificação dos profissionais com o tipo de atendimento realizado ${ }^{11}$. A afinidade com o trabalho em urgências foi indicada como motivação para atuar na UPA por $42,1 \%$ dos enfermeiros e por apenas $26,3 \%$ dos médicos, o que pode contribuir para a baixa expectativa desses profissionais de permanência na unidade.

\section{Conclusão}

A expansão acelerada nas UPA no Estado do Rio de Janeiro revelou limites na gestão do trabalho em saúde, expressas nas formas de contratação, fixação e no perfil dos profissionais nesses serviços. Apesar de diferenças iniciais entre as estratégias adotadas pelos governos estadual e municipais, houve no decorrer do tempo um efeito de difusão de experiências, com certa convergência no sentido de terceirizar a gestão e prestação de serviços das UPA para OS.
A contratação de profissionais por meio de OS permitiu a rápida abertura de unidades e flexibilidade na gestão do trabalho nas UPA. Porém, a terceirização não resolveu problemas de recrutamento e fixação, destacando-se nessas unidades a presença de profissionais jovens com limitada experiência em urgências e a rotatividade de médicos em áreas de vulnerabilidade social. Apesar da predominância de vínculos CLT, a instabilidade da inserção laboral associada à contratação por terceiros e a fragilização de carreiras públicas constituem desestímulos à permanência dos profissionais nas UPA, reforçando-as como postos de trabalho temporários.

As limitações de formação e as dificuldades de fixação de profissionais podem ter implicações para a qualidade do cuidado. Acrescente-se a isso o desafio de assegurar a continuidade da atenção, diante da natureza dos atendimentos de urgência e da fragilidade de instrumentos de articulação das UPA com outros serviços da rede.

Dificuldades de coordenação do sistema podem ser agravadas pela terceirização devido ao caráter temporário dos contratos, à rotatividade dos profissionais e gerentes e à diversificação das organizações prestadoras de serviços, que precisam se articular no território com outros prestadores privados e públicos, ligados a diferentes esferas de governo. A expansão desse modelo requer fortalecimento da capacidade estatal de monitoramento e avaliação do desempenho de cada unidade - cujos instrumentos ainda são limitados - e de regulação sistêmica, que considere as responsabilidades de atendimento e a inserção das UPA na rede de serviços.

Parte das dificuldades observadas reflete problemas mais amplos da gestão do trabalho em saúde no Brasil, associadas aos desafios do trabalho em urgências e às opções dos governos, inclusive relativas ao modelo das UPA e à terceirização. O enfrentamento dos desafios observados requer iniciativas articuladas de formação, regulação, incentivos e apoio profissional aos trabalhadores dos serviços públicos de saúde, como assinala Dal Poz 7, considerando ainda as peculiaridades da atenção às urgências.

Uma limitação da pesquisa foi que, apesar de a amostra das UPA visitadas e de coordenadores entrevistados ser representativa para o conjunto do estado, não foi possível adotar a mesma sistemática para seleção dos profissionais, optando-se por entrevistar aleatoriamente dois médicos e um enfermeiro em cada unidade. Além disso, a pesquisa não aprofundou temas relevantes para a gestão do trabalho, como formação profissional, educação permanente e processo de trabalho, a serem explorados em outros estudos. 
Pesquisas sobre o caráter e a pertinência desse tipo de unidade na rede de atenção em saúde, bem como sobre seu desempenho assistencial, também são necessárias à reorientação das políticas de gestão do trabalho e de atenção em urgências no SUS.

\section{Colaboradores}

C. V. Machado e L. D. Lima contribuíram com a concepção do artigo, levantamento e análise dos dados, redação do artigo e revisão final do artigo. G. O'Dwyer e T. W. F. Baptista contribuíram com o levantamento e análise dos dados, redação do artigo e revisão final do artigo. C. L. T. Andrade contribuiu com o levantamento, processamento estatístico e análise dos dados, elaboração de tabelas, redação da metodologia do artigo e revisão final do artigo. $R$. G. V. Pitthan contribuiu com o levantamento, processamento e análise de dados, elaboração de tabelas e figuras, redação de parte dos resultados e revisão final do artigo. $N$. Ibañez contribuiu com a concepção da pesquisa, análise dos dados, redação do artigo e revisão final do artigo.

\section{Agradecimentos}

A pesquisa foi financiada pelo Conselho Nacional de Secretários de Saúde (CONASS) e pelo Ministério da Saúde. C. V. Machado e L. D. Lima são bolsistas de produtividade em pesquisa do Conselho Nacional de Desenvolvimento Científico e Tecnológico (CNPq). Os autores agradecem ainda aos entrevistados da pesquisa e aos demais participantes do projeto Rede de Atenção às Urgências e Emergências: Avaliação da Implantação e Desempenho das Unidades de Pronto Atendimento (UPA). 


\section{Referências}

1. Narad RA. Coordination of the EMS system: an organizational theory approach. Prehosp Emerg Care 1998; 2:145-52.

2. Ministério da Saúde. Portaria MS/GM no 1600, de 07 de julho de 2011. Reformula a política nacional de atenção às urgências e institui a rede de atenção às urgências no Sistema Único de Saúde (SUS). Diário Oficial da Nação 2011; 8 jul.

3. Machado CV, Salvador FGF, O'Dwyer G. Serviço de Atendimento Móvel de Urgência: análise da política brasileira. Rev Saúde Pública 2011; 45:519-28.

4. O’Dwyer G, Konder MT, Machado CV, Alves CP, Alves RP. The current scenario of emergency care policies in Brazil. BMC Health Serv Res 2013; 13:70.

5. Pierantoni CR, Varella TC, Santos MR, França T, Garcia AC. Gestão do trabalho e da educação em saúde: recursos humanos em duas décadas do SUS. Physis (Rio J.) 2008; 18:685-704.

6. Machado MH, Oliveira E, Moyses N. Tendências do mercado de trabalho em saúde no Brasil. In: Pierantoni C, Dal Poz MR, França T, organizadores. O trabalho em saúde: abordagens quantitativas e qualitativas. Rio de Janeiro: Centro de Estudos e Pesquisa em Saúde Coletiva, Universidade do Estado do Rio de Janeiro; 2011. p. 103-16.

7. Dal Poz M. A crise da força de trabalho em saúde. Cad Saúde Pública 2013; 29:1924-6.

8. Martins MI, Molinaro A. Reestruturação produtiva e seu impacto nas relações de trabalho nos serviços públicos de saúde no Brasil. Ciênc Saúde Coletiva $2013 ; 18: 1667-76$.

9. Ministério da Administração Federal e Reforma do Estado. Organizações sociais. Cadernos MARE de Reforma do Estado 1997; (2).

10. Teixeira M, Martins MIC, Silva V. Novos desenhos institucionais e relações de trabalho no setor público de saúde no Brasil: as organizações sociais e as fundações estatais de direito privado. In: Martins MIC, Marques AP, Costa NR, Matos A, organizadores. Trabalho em saúde, desigualdades e políticas públicas. Braga: Editora Universidade do Minho; 2014. p. 100-23.

11. Deslandes SF. Frágeis deuses: profissionais da emergência entre os dados da violência e a recriação da vida. Rio de Janeiro: Editora Fiocruz; 2002.
12. Righi AW, Wachs P, Saurin TA. Characterizing complexity in socio-technical systems: a case study of a SAMU Medical Regulation Center. Work 2012; 41:1811-7.

13. Costa FL. Reforma do estado e contexto brasileiro: crítica do paradigma gerencialista. Rio de Janeiro: Fundação Getúlio Vargas; 2010.

14. Ibañez N, Bittar OJNV, Sá ENC, Yamamoto EK, Almeida MF, Castro CGJ. Organizações sociais de saúde: o modelo do Estado de São Paulo. Ciênc Saúde Coletiva 2001; 6:391-404.

15. Sano H, Abrucio FL. Promessas e resultados da nova gestão pública no Brasil: o caso das organizações sociais de São Paulo. Revista de Administração de Empresas 2008; 48:64-80.

16. Barbosa NB, Elias PEM. As organizações sociais de saúde como forma de gestão público/privado. Ciênc Saúde Coletiva 2010; 15:2483-95.

17. Machado CV. Novos modelos de gerência nos hospitais públicos: as experiências recentes. Physis (Rio J.) 2001; 11:105-97.

18. Instituto Brasileiro de Geografia e Estatística. Perfil dos estados brasileiros 2013 - pesquisa de informações básicas estaduais. Rio de Janeiro: Instituto Brasileiro de Geografia e Estatística; 2014.

19. Instituto Brasileiro de Geografia e Estatística. Perfil dos municípios brasileiros 2013 - pesquisa de informações básicas municipais. Rio de Janeiro: Instituto Brasileiro de Geografia e Estatística; 2014.

20. Harzheim E, Lima K, Hauser L. Pesquisa avaliativa sobre aspectos de implantação, estrutura, processo e resultados das Clínicas de Família na cidade do Rio de Janeiro [Relatório de Pesquisa]. Porto Alegre: Faculdade de Medicina, Universidade Federal do Rio Grande do Sul; 2013.

21. Giglio-Jacquemot A. Urgências e emergências em saúde: perspectivas de profissionais e usuários. Rio de Janeiro: Editora Fiocruz; 2005.

22. Haddad AE, Moritall MC, Pierantoni CR, Brenelli SL, Passarella T, Campos FE. Formação de profissionais de saúde no Brasil: uma análise no período de 1991 a 2008. Rev Saúde Pública 2010; 44:383-93.

23. Ceccim RB, Pinto LF. A formação e especialização de profissionais de saúde e a necessidade política de enfrentar as desigualdades sociais e regionais. Rev Bras Educ Méd 2007; 31:266-77. 


\section{Abstract}

In the late 2000s, the expansion of Emergency Care Units (UPAs) in Brazil's policy for provision of urgent healthcare included hiring a large contingent of health professionals. This article analyzes government strategies for workforce management and the profile of these professionals in the UPAs in the State of Rio de Janeiro, which has the largest number of such units in the country. The methods included document analysis, interviews with managers, and visits to the UPAs and interviews with coordinators, physicians, and nurses. The results showed that the workforce management strategies varied over time and according to administrative sphere (state versus municipal). The so-called Social Organizations became the main hirers of health professionals in the UPAs, since they allowed management flexibility. However, there were problems with selection and stability, with a predominance of young professionals with limited experience and high physician turnover. Instability associated with outsourced hiring reinforced the view of work at the UPA as a temporary job.

Emergencies; Health Personnel; Health Management; Health Policy, Planning and Management

\section{Resumen}

Al final del año 2000, la expansión de las unidades de atención de urgencias (UPA por sus siglas en portugués) en el ámbito de la política brasileña de atención a las urgencias implicó la contratación de muchos profesionales. El artículo analiza las estrategias de gestión del trabajo de los gobiernos y el perfil de los profesionales de las UPAs en el estado de Río de Janeiro, que tiene el mayor número de unidades en el país. Los métodos comprendieron: análisis documental; entrevistas con gestores; visitas a las UPAs con administración de cuestionarios a coordinadores, médicos y enfermeros. Los resultados evidenciaron que las estrategias de gestión del trabajo variaron según la esfera administrativa (estatal o municipal) y a lo largo del tiempo. Las organizaciones sociales se convirtieron en las principales contratantes de profesionales en las UPA por propiciar flexibilidad en la gerencia. No obstante, se hicieron explícitos problemas de selección y fijación, como la predominancia de profesionales jóvenes con poca experiencia y la rotatividad de médicos. La inestabilidad asociada a la contratación por terceros reforzó la visión de la UPA como un trabajo temporal.

Urgencias Médicas; Personal de Salud; Gestión en Salud; Políticas, Planificación y Administración en Salud
Recebido em 15/Nov/2014

Versão final reapresentada em 18/Jun/2015 Aprovado em 26/Jun/2015 